\title{
Original Article \\ Exploring the Evidence Base for Acupuncture in the Treatment of Ménière's Syndrome—A Systematic Review
}

\author{
Andrew F. Long, ${ }^{1}$ Mei Xing, ${ }^{2}$ Ken Morgan, ${ }^{3}$ and Alison Brettle ${ }^{4}$ \\ ${ }^{1}$ School of Healthcare, University of Leeds, Leeds, LS2 9UT, UK \\ ${ }^{2}$ School of Community Health Sciences and Social Care, University of Salford, Salford, UK \\ ${ }^{3}$ Acupuncturist, Bolton, UK \\ ${ }^{4}$ School of Nursing, University of Salford, Salford, UK \\ Correspondence should be addressed to Andrew F. Long, a.f.long@leeds.ac.uk
}

Received 24 September 2008; Accepted 5 May 2009

Copyright (C) 2011 Andrew F. Long et al. This is an open access article distributed under the Creative Commons Attribution License, which permits unrestricted use, distribution, and reproduction in any medium, provided the original work is properly cited.

\begin{abstract}
Ménière's syndrome is a long-term, progressive disease that damages the balance and hearing parts of the inner ear. To address the paucity of information on which evidence-based treatment decisions should be made, a systematic review of acupuncture for Ménière's syndrome was undertaken. The method used was a systematic review of English and Chinese literature, from six databases for randomized, non-randomized and observational studies. All studies were critically appraised and a narrative approach to data synthesis was adopted. Twenty-seven studies were included in this review (9 in English and 18 in Chinese languages): three randomized controlled trials, three non-randomized controlled studies and four pre-test, post-test designs. All but one of the studies was conducted in China. The studies covered body acupuncture, ear acupuncture, scalp acupuncture, fluid acupuncture point injection and moxibustion. The studies were of varying quality. The weight of evidence, across all study types, is of beneficial effect from acupuncture, for those in an acute phase or those who have had Ménière's syndrome for a number of years. The review reinforces the importance of searching for studies from English and Chinese literature. The transferability of the findings from China to a Western context needs confirmation. Further research is also needed to clarify questions around the appropriate frequency and number of treatment/courses of acupuncture. The weight of evidence suggests a potential benefit of acupuncture for persons with Ménière's disease, including those in an acute phase and reinforces the importance of searching for published studies in the Chinese language.
\end{abstract}

\section{Introduction}

Ménière's syndrome is a long-term, progressive disease that damages the balance and hearing parts of the inner ear. It is most common between the ages of 40 and 50 and rare in children and onset after the age of 60 . Its incidence is about 1 in 1000, equally distributed between men and women and well documented in Caucasian, African-American and Asian races [1]. The etiology and treatment of Ménière's disease is not fully understood. In a literature-based, clinical review of the diagnosis and treatment of Ménière's disease, Saeed [2] commented that "currently, the treatment of Ménière's disease is empirical. As yet, no treatment has prospectively modified the clinical course of the condition and thereby prevented the progressive hearing loss." Indeed, Thorp et al. [3] in a critical review of studies of medical and surgical approaches queried whether any evidence-based medicine existed in the treatment of Ménière's disease.

Conventional biomedical treatments include drugs, diet and surgery. Drugs often form the first line of treatment. These include: diuretics (to reduce the amount of sodium in the body); drugs to block symptoms of motion sickness, nausea and vomiting, and anxiety and vertigo; systemic or local corticosteroids to reduce inflammation within the inner ear and to stop any immune reactions; antibiotics; and drugs to improve blood flow in the inner ear [4]. The most commonly recommended dietary treatment is a low sodium diet. Patients have sometimes been helped by limiting certain components of their diet such as sugar, monosodium glutamate, caffeine and alcohol [4]. Surgical treatment is the last resort of conventional medicine for Ménière's disease, the most controversial [2] and of greatest risk. 
TABLE 1: Inclusion and exclusion criteria.

Inclusion criteria:

Study on patients with a diagnosis of Ménière's disease

All types of TCM acupuncture or moxibustion

Study using acupuncture or moxibustion in addition to other TCM, for example, herbal medicine

Studies of any controlled type and case series if the sample size was $\geq 10$

Studies in the English or Chinese languages

Exclusion criteria:

Studies with a sample size of $<10$ or single case reports or reports of opinion

Studies which treated dizziness, tinnitus or other symptoms of Ménière's disease without a diagnosis of Ménière's

Other forms of Traditional Chinese Medicine without acupuncture or moxibustion

As with other chronic diseases where treatment may have serious side-effects [5], or is variably experienced by patients as beneficial [6], or as with Ménière's disease there is limited high quality evidence as to the most appropriate treatments [3], some who suffer from the condition may explore the potential of complementary and alternative medicine (CAM) therapies to alleviate their symptoms. However, there is little published information on which CAM therapies might help those with Ménière's disease. To begin to address this evidence gap, and following anecdotal comments about the potential benefits of one CAM therapy-traditional Chinese medicine (TCM) acupuncture-a systematic review was undertaken of published evidence on the effectiveness of acupuncture in treating the symptoms of Ménière's disease, drawing on both English and Chinese language sources. This article reports the findings from that review.

\section{Methods}

2.1. Searching. As there was no previous review of published evidence in this area, and thus to ensure comprehensive coverage of the literature, any published English or Chinese language study, which used a quantitative outcome measure and had a sample size of 10 or more, was included. The criterion for sample size was set low in anticipation that current literature in this field might have a relatively small sample size; an a priori prescribed higher limit might unduly restrict the located evidence. The search was undertaken in two parts. The first was based on a search of English language literature, undertaken in 2004/-5 and forming the focus for a final year dissertation of an undergraduate student (K.M.) studying for a BSc in Traditional Chinese Medicine (Acupuncture) at the University of Salford; this was updated in 2009. The second comprised an opportunistic search of Chinese language literature as part of a study visit of one of the authors (M.X.) to China in the winter of 2003. The inclusion and exclusion criteria are summarized in Table 1.

Six databases were searched: MEDLINE via OVID interface (1966 to January 2009); The Cochrane Controlled Trials Register (on 18 January 2009), EMBASE (1974 to
January 2009); CINAHL (1982 to January 2009); AMED (1985 to January 2009); and, for the Chinese language review, the Chinese database of Science and Technology (1993 to August 2003). A search of eCAM was also undertaken (on 6 January 2009). Hand searching of Chinese journals related to acupuncture and moxibustion was undertaken in China for the months of September to December 2003. Search terms of the electronic databases focused on terms related to acupuncture and Ménière's disease. Free text search terms were used and controlled subject headings were exploded so that any subcategories were included (e.g., endolymphatic hydrops, acupuncture therapy or electroacupuncture). See the appendix for an illustrative search strategy.

2.2. Appraisal and Analysis. Titles and abstracts were screened by one member (K.M. or M.X., respectively) and 27 papers were included in the review (Figure 1). All included studies were critically appraised. For the English language studies, an established evaluation tool [7] was used, suitably adapted to add in appropriate search-specific and acupuncture-related aspects [8]. For the Chinese language literature, data were extracted and simultaneously translated into English, using a standard template which included the following categories: study design; sample; treatment method; duration of treatment; outcome measurement; results; commentary on the appropriateness of acupuncture; and, overall comments/critique of the paper. Each English language paper was read and appraised by two members of the team independently (K.M. and M.X./A.F.L.) and any discrepancies resolved through discussion. The Chinese summaries were critically appraised (M.X. and A.F.L.) and where necessary further data extracted from the primary source.

A narrative approach to data synthesis was taken, with greater weight given to studies of greatest quality (minimizing bias, accuracy of diagnosis, appropriateness of acupuncture treatment and use of appropriate outcome assessment). A meta-analysis was inappropriate due to difference in study designs and outcome measures and the limited amount of quantitative data for individual patients. Restriction of the analysis to only controlled trials would have substantially narrowed the available evidence base and was judged inappropriate in light of the lack of previous attempts to draw together evidence on acupuncture for Ménière's disease.

\section{Results}

3.1. Nature of the Evidence. Of the 27 studies included in the review, 9 were studies in the English language and 18 in the Chinese language. All but one of the studies was conducted in China. The studies comprised: three randomized controlled trials (RCTs) [9-11]; three non-randomized controlled studies [12-14]; four pre-test, post-test designs [15-18]; nine post-test designs [19-27] and eight case series reports [28-35]. Together they comprised a total of 1888 patients in the acupuncture treatment arm. Seventeen of the studies followed the patients up for at least a year after the end of treatment.

Particular symptoms formed the focus of a number of studies, including vertigo [21] and dizziness [11, 18, 32]. 


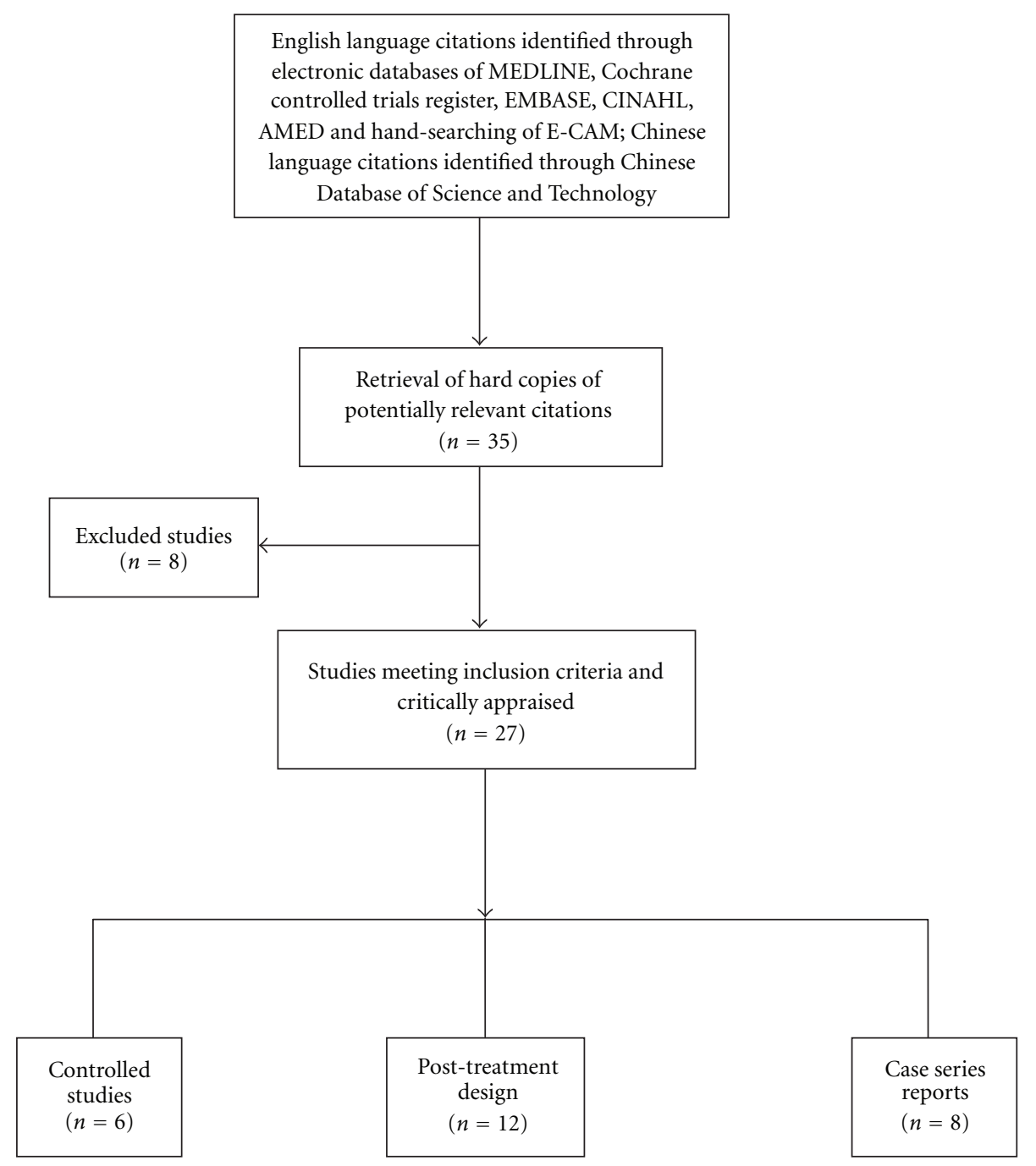

FIGURE 1: Flowchart literature search process.

The patients had been suffering from Ménière's disease for various periods of time, ranging from 1 to 24 years. Thirteen of the studies included patients at an acute phase, within 110 days of an acute attack. Three studies $[9,15,30]$ explicitly focused on the effects of acupuncture for acute symptom relief. Sample sizes varied from 15 [11] to 286 [33], and 5 had a sample size of less than $30[18,19,25,26,30]$. The six controlled studies had total sample sizes ranging from 36 to 189 in the acupuncture arm. Nine of the studies had 50 or fewer participants.

The majority of studies employed a graded outcome measurement approach, differentiating three to four categories: "cured", "outstandingly effective", "effective/improved" or "not effective". "Cured" had the common meaning of "dizziness and other symptoms having disappeared" or "all symptoms disappeared", "able to return to work/resume normal activities" and "no recurrence within a 1 or 2 year period" (depending on the study's follow-up time). "Outstandingly effective" had a similar meaning but with recurrence of symptoms "occasionally", by, for example, 6 months.
"Effective/improved" related to "relief of symptoms". Particular symptoms were often mentioned as part of the achieved outcomes, in particular dizziness and vertigo.

3.2. Nature of Acupuncture. The studies covered five types of acupuncture: body acupuncture; ear acupuncture; scalp acupuncture; fluid acupuncture point injection; and moxibustion. All such types are classifiable as falling within the TCM style, ensuring the "de-qi" sensation. Half of the studies involved an individualized TCM prescription approach to the treatment and half used a pre-set prescription, itself based on TCM principles and approaches to treating particular symptoms commonly experienced by persons with Ménière's disease, in particular, symptoms of dizziness, vertigo, nausea and vomiting. While none of studies mentioned the background of the acupuncturist, acupuncturists in China are all qualified medical doctors and at least 4 years training is required to work in a hospital.

No single, uniform number of courses or duration of treatment within each course was apparent. In two controlled 
studies $[10,14]$, once daily acupuncture was undertaken for 10 days; in another [13], no indication of the number of treatments or how often the treatment was given; in yet another [30], patients continued with the treatment until symptom relief. Across the studies, a treatment "once a day" for a course of "up to 10 sessions" with the possibility of a second (or more) course was common.

3.3. Quality of the Evidence. A number of weaknesses were evident in the studies. First, as only six of the studies had a comparison group and four others also included pre-treatment measurement, any observed improved outcomes could have arisen because of other factors, including symptom remission. Secondly, only eight of the studies either included audiometric testing $[9,19,21]$ or recruited Ménière's disease patients according to established criteria for Ménière's [10, 18, 23, 24, 26]. Only two studies included audiometric testing as part of their outcome measurement $[9,27]$. Thirdly, most of the Chinese language studies did not provide detail on the inclusion/exclusion criteria. Fourthly, very limited detail was provided on the choice of study participants or setting of the studies. Fifthly, the length of follow-up (and thus final outcome measurement point) varied; eight studies had a follow-up time of 2 years and nine had 1 year, while for seven no or little detail was provided.

3.4. Evidence of Effectiveness. The weight of evidence, across all study types, is one of the beneficial effects from five types of acupuncture-body, ear or scalp acupuncture, fluid acupuncture point injection, or moxibustion (Table 2 ). The three randomized controlled trials [9-11] demonstrate a statistically significant benefit of body or scalp acupuncture against Western medicine and vitamins, with a mean difference in the total effectiveness percentage of $14 \%$ in favor of acupuncture $(P<.01)$. Two non-randomized controlled trials, comparing body acupuncture against Western medicine [12] or Chinese herbal medicine [13], show a similar mean difference in total effectiveness percentage (19\%, $P<.001)$. The evidence from the four pre-test, posttest studies reports even higher success rates (90-100\%) for acupuncture, including moxibustion on acupoint Du-20, either on its own [18] or combined with body [15], and/or ear acupuncture [16] or herbal medicine [17]. High success rates are also reported from the post-test and case-series studies. As 13 studies included patients in an acute phase of Ménière's disease, the evidence suggests beneficial effects for both those in an acute phase and who have had Ménière's for a number of years. There is, however, insufficient evidence to recommend one, or another, type of acupuncture, nor the number of courses of treatment that might be needed for beneficial effect.

\section{Discussion}

This is the first systematic review of evidence on the potential benefits of acupuncture for the treatment of Ménière's disease and draws on both English and Chinese language sources. It demonstrates beneficial effects from up to five types of acupuncture (body, ear or scalp acupuncture, fluid acupuncture point injection, or moxibustion) and for both those in an acute phase and who have had Ménière's disease for a number of years. At the same time, it is important to note that the quality of evidence varies and is drawn from studies with varying potentiality for bias. In the five studies with a non-acupuncture comparison group (irrespective of the mode of allocation), the differences in total effectiveness percentage in favor of acupuncture were of the order of $14-19 \%$.

Given that the weight of evidence is from China, further research within a Western context is required. There is also a need to clarify questions around the appropriate frequency and number of treatments or courses of acupuncture. This is especially important where persons have to pay for acupuncture treatment. Exploring possible sets of predefined acupuncture points is also important, given that not all acupuncturists in the UK or Europe adopt a TCM diagnosis and treatment approach [36].

The review differs in relation to others in the CAM area, as it draws on both English and Chinese literature. Such an approach led to the inclusion of 18 additional studies and, in principle (irrespective of study quality), strengthens the conclusions of the review. A similar conclusion is drawn by Yuan et al. [37] who explored treatment regimens of acupuncture for low back pain, accessing English and Chinese literature, the latter located by Chinese experts. Indeed, their systematic review identified substantive differences in the treatment frequency between practice in China and the West.

The inclusion of English and Chinese language papers does not however avoid the issue of publication bias; publications in other languages would still be excluded. Neither does it avoid publication bias arising from the greater likelihood of the reporting of positive results (i.e., the nonreporting of negative studies). As Moher et al. [38] argued, substantial bias may occur in the results of a CAM systematic review if languages other than English are excluded. Extending the searching process to the Chinese language is an important step, given the lengthy historical tradition and use of acupuncture within China. Indeed, accessing research literature published in the Chinese language perhaps needs to become a sine qua non in this type of literature review. This is challenging, requiring access to Chinese language readers $[39,40]$. It was possible in this study only because one of the project team members (M.X.) was native Chinese. Optimal methods of accessing and including Chinese language literature however need further exploration.

Generalizing or transferring the results of studies undertaken in another country and cultural context is not straightforward. All but one of the studies took place in China. Citizens' attitudes to TCM and acupuncture in a Chinese cultural context, where there is a long tradition, recognition and establishment within the health care sector [36], along with established education and training approaches, are likely to be very different than in a Western European context. This has important consequences for patients deciding when to seek acupuncture. In China, acupuncture may be sought early or as a routine treatment. In the West, where the first line of treatment for someone with Ménière's 


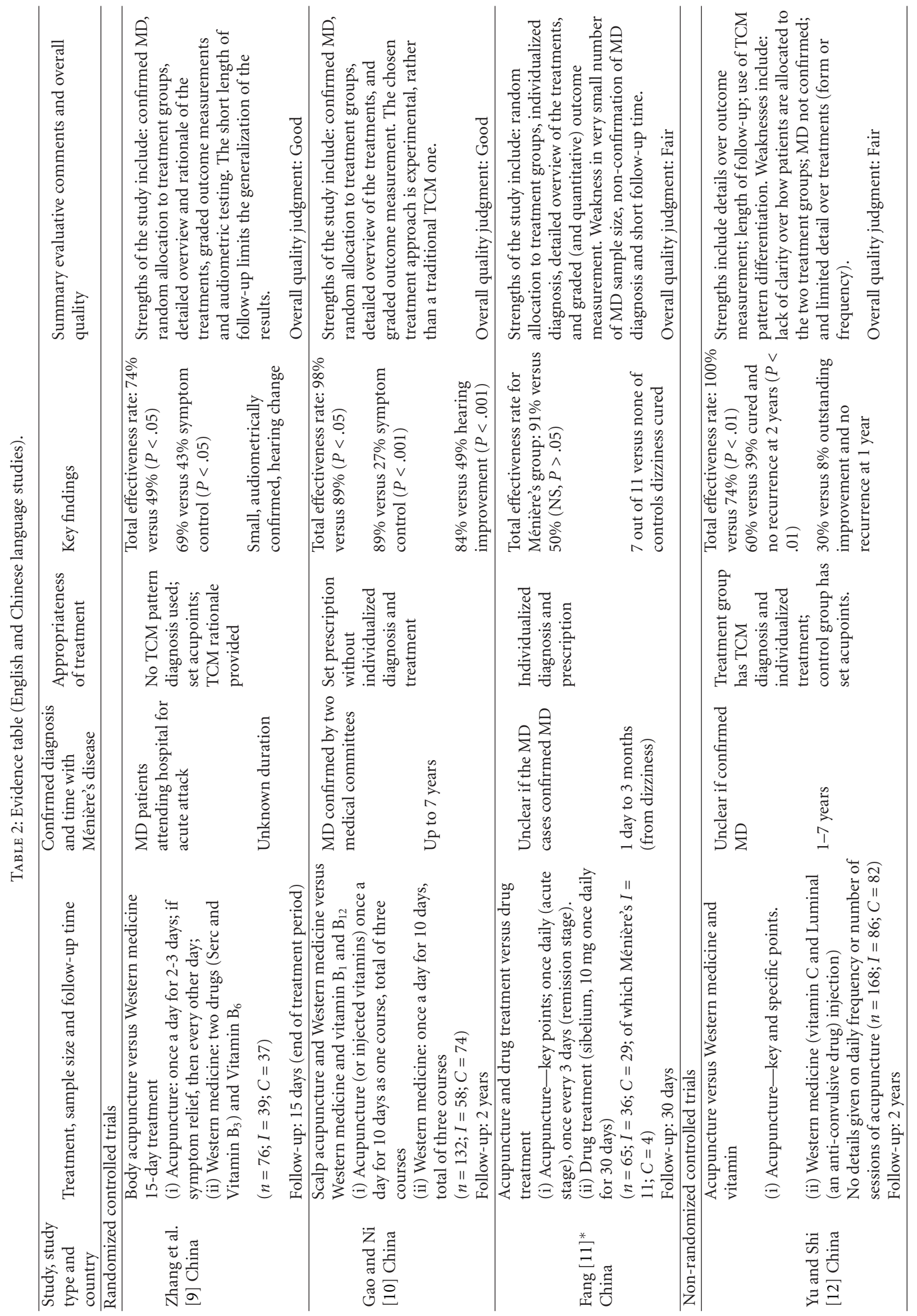




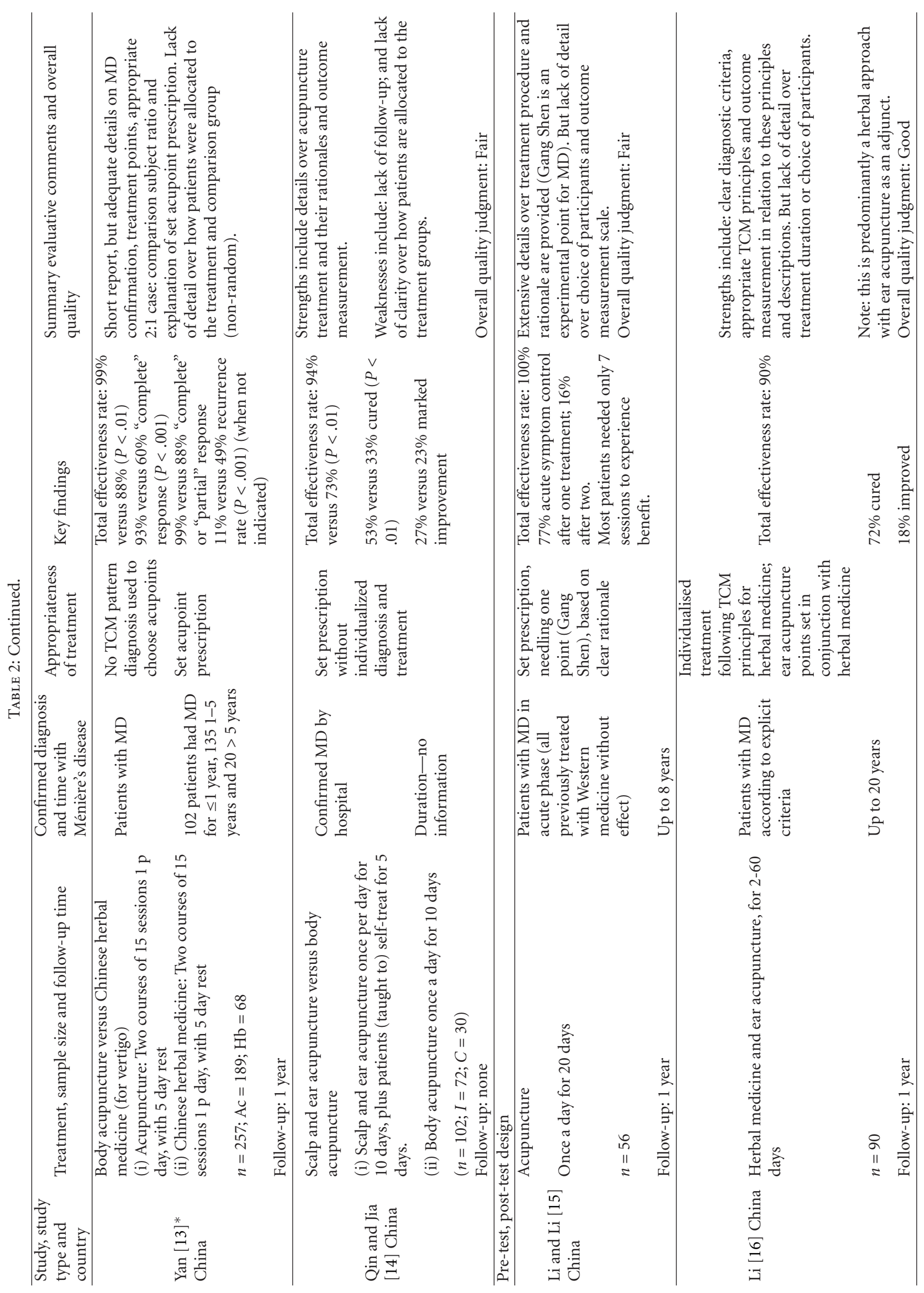




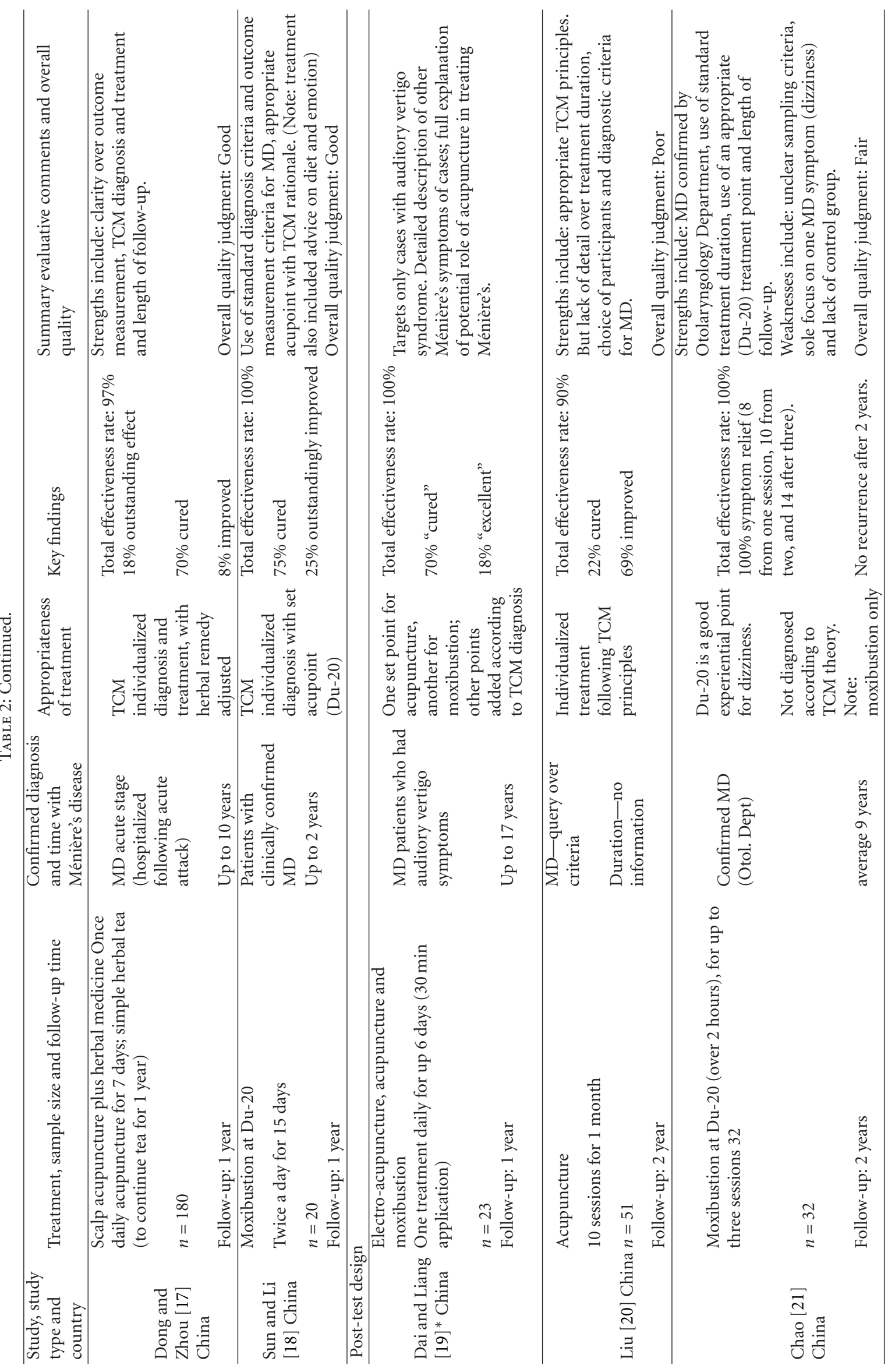




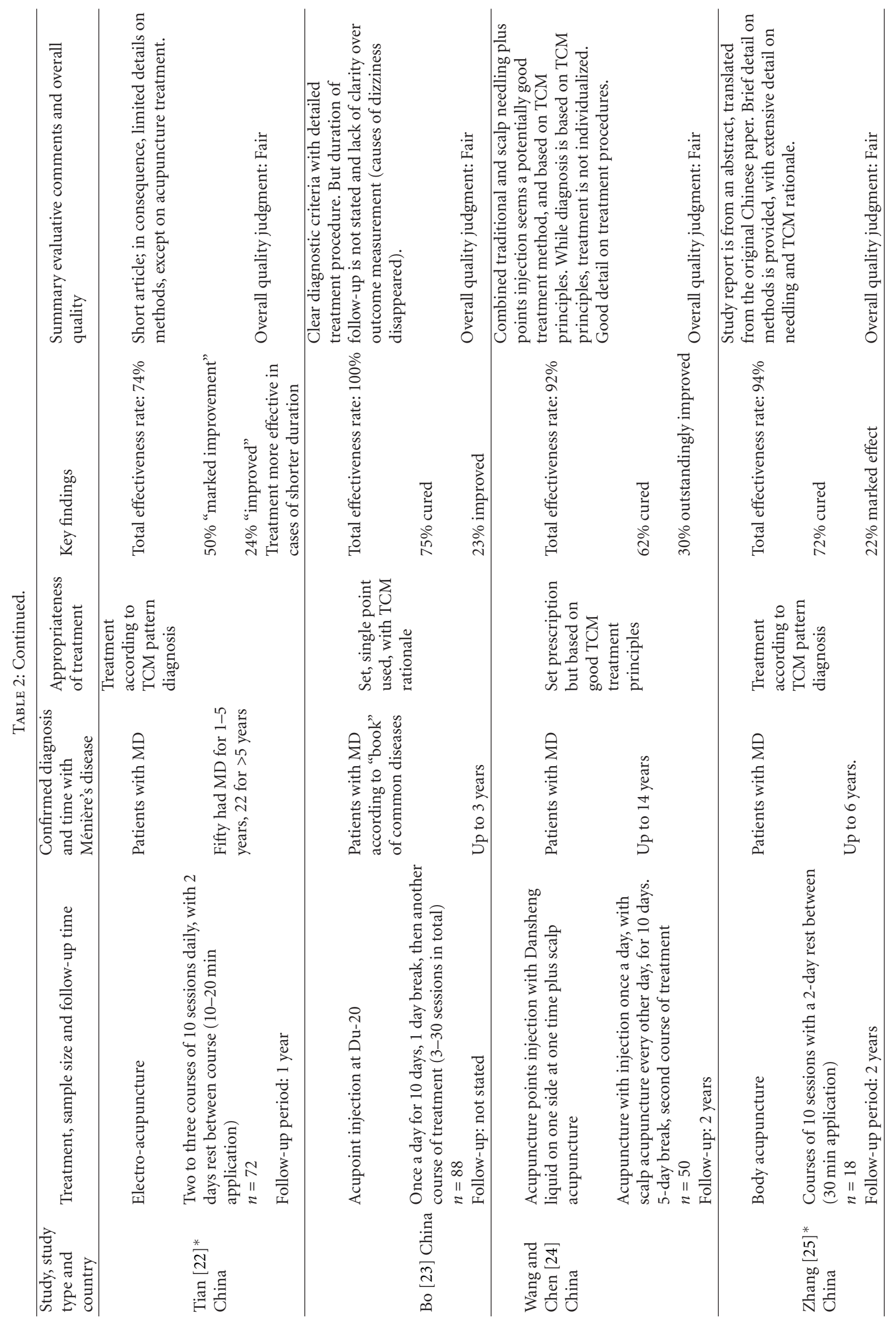




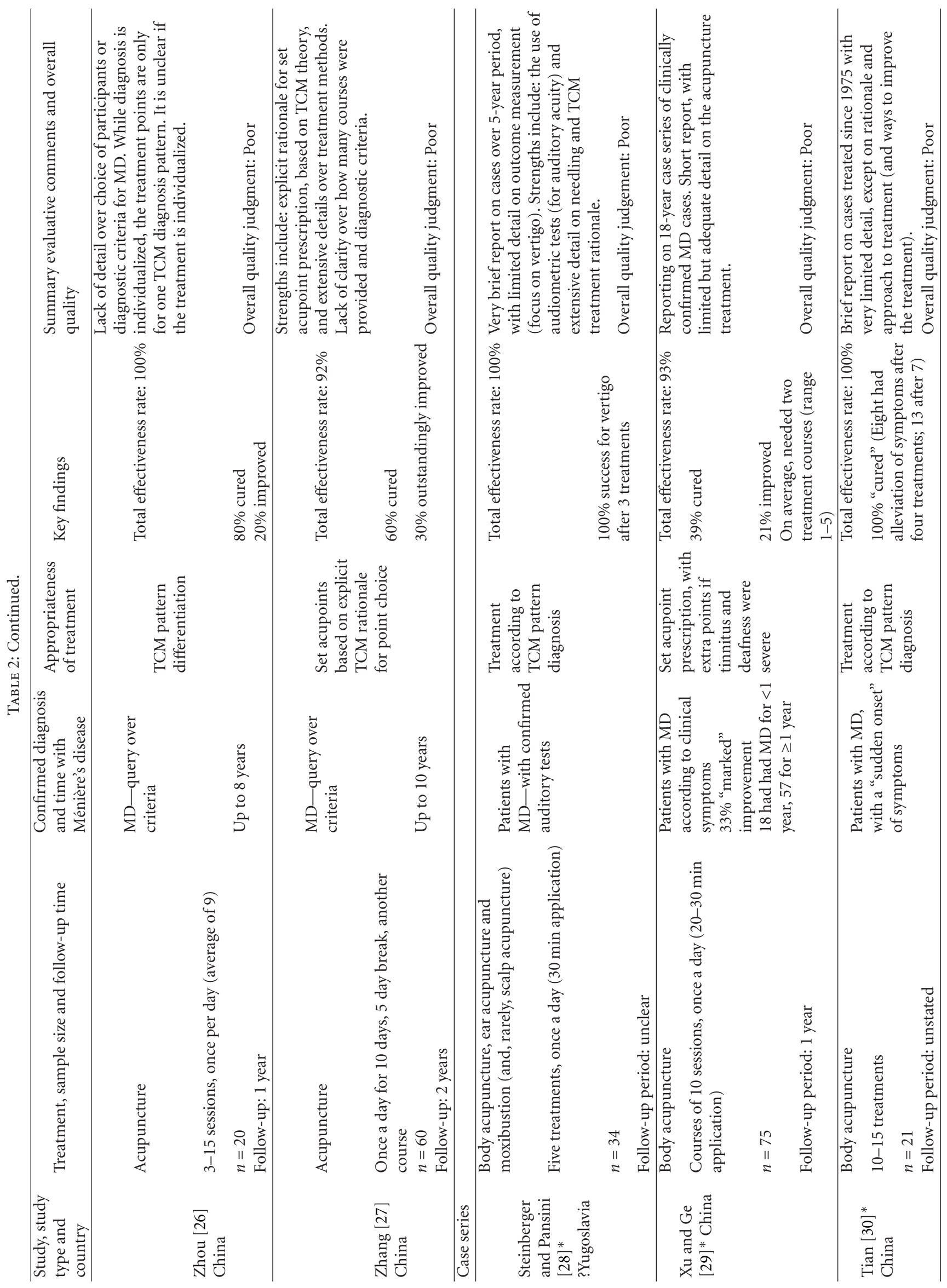




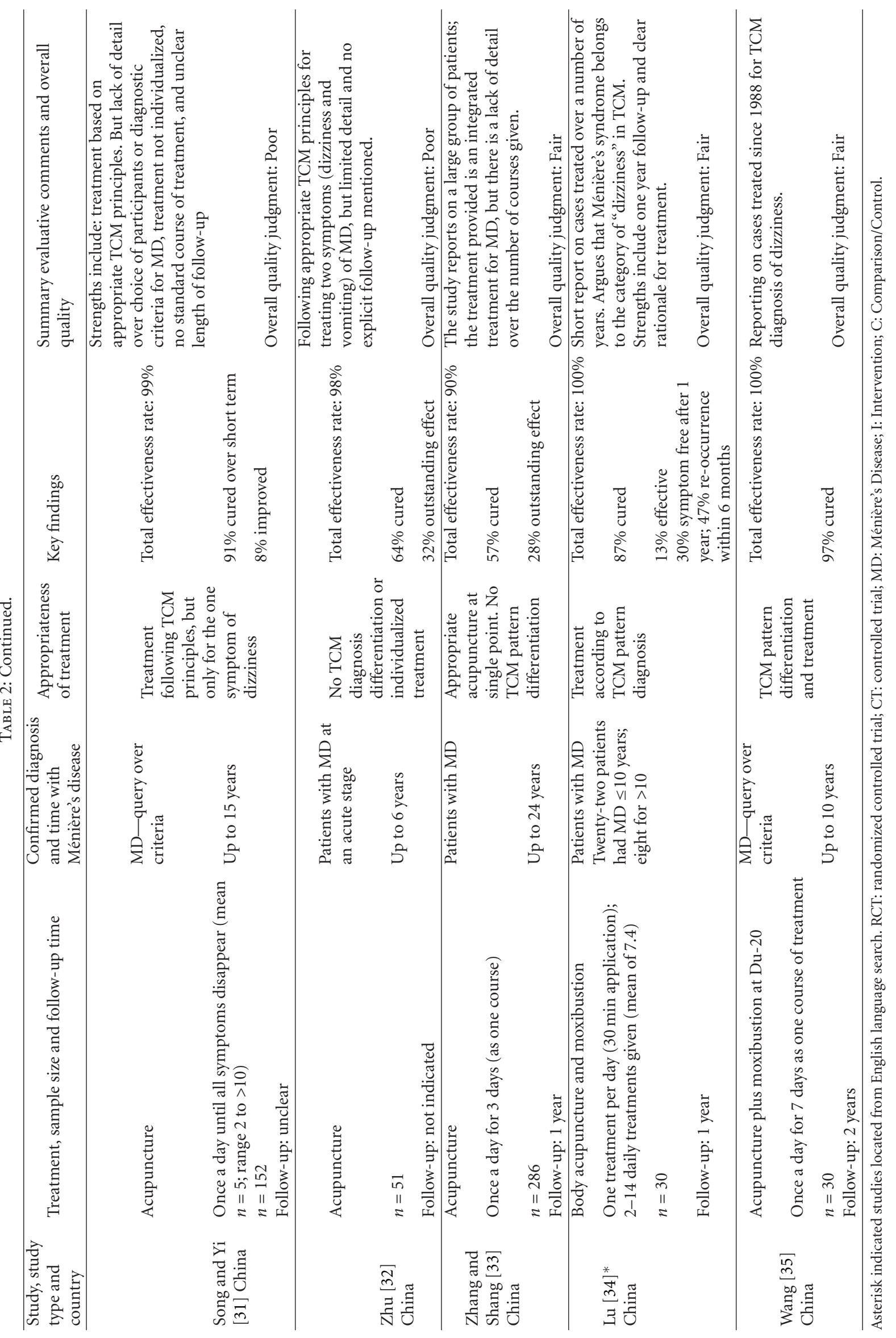


disease would likely be biomedical, acupuncture may be sought only when the condition becomes chronic. There are also differences in education and training of, and access to, acupuncturists (traditional TCM trained practitioners or Western acupuncture [41], thus the recognized importance of reporting studies using the STRICTA framework [8]. This strongly suggests the need for a controlled trial of acupuncture, for both persons in an acute phase and those who have had Ménière's disease for a number of years.

Any such study needs to be appropriately designed. As there is no definitive, demonstrated bio-medical curative approach for Ménière's disease, choice of comparison group is not straightforward. A placebo design would seem both inappropriate and ethically problematic, as it is highly improbable that potential study participants will not be taking some medication or other approach to enable them to cope. While arguing for the need for greater and more rigorous evidence, Thorp et al. [3] make a similar comment in the context of conventional medicine. In Europe, an appropriate comparison might be drug treatment. In China, an appropriate contrast might be a (Western) drug approach or different forms of TCM approaches (e.g., types of acupuncture and/or herbal medicine). Indeed, the former form of comparison (acupuncture versus a drug or herbal approach) was used in four of the included controlled studies [9-12], and the latter (different forms of acupuncture or Chinese herbal medicine) in two others $[13,14]$. It is important to note that choice of comparison group should be culturally determined.

Whatever choice is made, it is important that full methodological details are provided in the research report, utilizing the STRICTA recommendations [8] both as a guide to reporting but also study design. The review suggests the potential of exploring treatment "once a day" for a course of "up to 10 sessions" with the possibility of a second (or more) course. Such research should measure both the shortand longer-term symptom effect, based upon patient reports of symptom benefit and (time before any) recurrence and extent of severity/symptom relief at any recurrence, general health and well-being effects and a potential greater resolve to cope with the illness.

There are some limitations to this review. First, the Chinese literature search accessed only one database and limited hand searching. More studies might be generated from a wider search. Secondly, it is notable that the studies from China report positive effects of acupuncture. This may be due to publication bias, [42] or be a true effect, demonstrating that frequent and a large number of treatment sessions, evident in the Chinese studies, leads to better outcomes [37]. In this regard it is interesting to note one of the findings from a systematic review of clinical trials on acupuncture in the Japanese literature [43]; studies undertaken pre-1990 tended to focus on the appropriate choice of acupuncture technique, whereas post-1990 the focus tended to be on evaluation of acupuncture efficacy. This finding has some resonance within the Chinese literature in this review, especially within the case-series studies and their presentation and the depth of explanation about technique. Thirdly, inclusion of nonrandomized and uncontrolled studies in the review could be argued to be problematic [44]. Such studies were used here as a means to support, or otherwise, findings from more powerful studies. In addition, such studies provided additional information on the maintenance of beneficial effects. Fourthly, in a recent report relating specifically to Cochrane systematic reviews of TCM, where only 5 of the 28 described RCTs could be authenticated as RCTs, Wu et al. [45] argue for the need for review authors to verify with study authors for claims that their RCTs were in fact randomized. This was not undertaken in this study. Fifthly, no attempt is made to draw together any of the TCM explanations indicated in the included papers about the mechanisms that might underpin acupuncture effecting the symptoms of Ménière's disease or other factors which may be associated with such symptoms.

\section{Conclusion}

The aim of this systematic review was to locate and critically appraise evidence for acupuncture as a treatment for Ménière's disease, drawing from both English and Chinese language literature. Despite the range in the quality of the located evidence, the overall conclusion is of the potential benefit of acupuncture for persons with Ménière's disease, including those in an acute phase. The review also demonstrates the importance of searching for studies in the Chinese language for such a therapy as acupuncture, given its lengthy historical tradition within Chinese medicine. As all but one of the studies took place within China, further research is needed in a Western health care context and to examine the frequency and number of treatment or courses of acupuncture.

\section{Appendix}

\section{Search Strategy for MEDLINE via OVID interface}

(1) meniere\$.mp.

(2) (endolymphatic adj hydrops).mp.

(3) exp Endolymphatic Hydrops/

(4) exp Meniere Disease/

(5) 1 or 2 or 3 or 4

(6) exp Acupuncture Therapy/

(7) (acupuncture or electro? acupuncture or auriculotherapy or (auricular adj needles) or (needling adj therapy) or (electric adj needling)).mp.

(8) exp Medicine, Chinese Traditional/

(9) 6 or 7 or 8

(10) 9 and 5

Key:

$\mathrm{mp}=$ title, original title, abstract, name of substance word, subject heading word

exp $=$ explode subject heading 


$$
\begin{aligned}
/ & =\text { subject heading }(\mathrm{MESH}) \text { search } \\
\$ & =\text { truncation symbol } \\
? & =\text { wildcard symbol } \\
\text { adj } & =\text { adjacent to }
\end{aligned}
$$

\section{Funding}

Research Development Fund of the University of Salford.

\section{Acknowledgements}

The authors gratefully acknowledge the comments of Tony Bennett, as expert patient.

\section{References}

[1] M. Ferrari and PDxMD, PDXMD Ear, Nose \& Throat Disorders, Elsevier Science, Philadelphia, Pa, USA, 2003.

[2] S. R. Saeed, "Diagnosis and treatment of Ménière's disease," British Medical Journal, vol. 316, no. 7128, pp. 368-372, 1998.

[3] M. A. Thorp, Z. P. Shehab, M. L. Bance, and J. A. Rutka, "Does evidence-based medicine exist in the treatment of Ménière's disease? A critical review of the last decade of publications," Clinical Otolaryngology and Allied Sciences, vol. 25, no. 6, pp. 456-460, 2000.

[4] A. Salt, Compilation of Treatments for Ménière's Disease, Washington University Cochlear Fluids Research Laboratory, St. Louis, Mo, USA, 2004, http://oto2.wustl.edu/men/mntreat .htm.

[5] C. Vincent and A. Furnham, "Why do patients turn to complementary medicine? An empirical study," British Journal of Clinical Psychology, vol. 35, no. 1, pp. 37-48, 1996.

[6] S. Thorne, B. Paterson, C. Russell, and A. Schultz, "Complementary/alternative medicine in chronic illness as informed self-care decision making," International Journal of Nursing Studies, vol. 39, no. 7, pp. 671-683, 2002.

[7] J. S. Reisch, J. E. Tyson, and S. G. Mize, "Aid to the evaluation of therapeutic studies," Pediatrics, vol. 84, no. 5, pp. 815-827, 1989.

[8] H. MacPherson, A. White, M. Cummings, K. Jobst, K. Rose, and R. Niemtzow, "Standards for reporting interventions in controlled trials of acupuncture: the STRICTA recommendations," Acupuncture in Medicine, vol. 20, no. 1, pp. 22-25, 2002.

[9] Z. F. Zhang, F. Xue, Z. D. He, S. Hou, and H. Li, "Clinical study on acupuncture and moxibustion for treatment of acute onset stage of Ménière's syndrome," Shanghai Journal of Acupuncture and Moxibustion, vol. 2, pp. 28-31, 1983.

[10] X. P. Gao and H. H. Ni, "Observation on therapeutic effect of scalp-acupuncture on Ménière's disease," Chinese Acupuncture and Moxibustion, vol. 22, pp. 583-584, 2002.

[11] G. Fang, "Observation on treatment of dizziness mainly by acupuncture," Journal of Traditional Chinese Medicine, vol. 27, no. 1, pp. 16-18, 2007.

[12] S. Yu and X. Shi, "Acupuncture for the treatment of 86 cases of Ménière's syndrome," Shanghai Acupuncture Journal, vol. 16, p. 21, 2002.

[13] S. M. Yan, "Acupuncture for Ménière's syndrome: short- and long-term observation of 189 cases," International Journal of Clinical Acupuncture, vol. 10, pp. 303-304, 1999.

[14] Y. X. Qin and X. C. Jia, "Combination of scalp acupuncture and ear acupuncture for the treatment of 78 cases of Ménière's syndromes," Journal of Chinese Modern Medicine, vol. 6, pp. 52-53, 2003.
[15] C. X. Li and C. Z. Li, "Clinical observation of acupuncture for the treatment of 56 cases of Ménière's syndrome," J Acupunct Clin Pract, vol. 9, p. 10, 1993.

[16] X. H. Li, "Acupuncture in combination of herbal medicine for the treatment of 90 Ménière's syndrome," Liaoning Journal of Traditional Chinese Medicine, vol. 26, p. 220, 1999.

[17] J. J. Dong and Z. Zhou, "Dizziness decoction combine with scalp acupuncture for the treatment of 180 cases of Ménière's syndrome," Shanxi Journal of Traditional Chinese Medicine, vol. 22, pp. 470-471, 2001.

[18] L. J. Sun and A. X. Li, "Pressing moxibustion on D20 for the treatment of Ménière's syndrome," Chinese Acupuncture and Moxibustion, vol. 21, p. 10, 2001.

[19] J. Dai and S. Liang, "Treatment of Ménière's disease by needling Ear-Shenmen and moxibustion at Baihui," International Journal of Clinical Acupuncture, vol. 4, pp. 281-284, 1993.

[20] Y. Liu, "Acupuncture for the treatment of 51 cases of Ménière's syndrome," Shanghai Journal of Acupuncture, vol. 14, p. 258, 1995.

[21] L. Y. Chao, "Moxibustion on Du-20 for the treatment of 32 cases of Ménière's syndrome," Xing Jiang J Chin Med, vol. 15, p. 28, 1996.

[22] Z. M. Tian, “Treatment by electro-acupuncture-Ménière's disease," Journal of Chinese Medicine, vol. 61, pp. 28-29, 1999.

[23] S. L. Bo, "Injecting D20 for the treatment of 88 cases of Ménière's syndrome," Chinese Acupuncture and Moxibustion, vol. 22, p. 72, 2002.

[24] Q. Q. Wang and H. L. Chen, "Combination of acupuncture points injection with scalp acupuncture for the treatment of Ménière's syndrome," Journal of Sichuan of Traditional Chinese Medicine, vol. 20, p. 73, 2002.

[25] Z. Zhang, "The treatment of 18 cases of Ménière's disease mainly with the points Bai Hui (GV-20) joined to Hou Ding (GV-19). Ji Lin Zhong Yi Yao (Jilin Chin Med Medicinals) 2002; 9: 48. Précis in English, Wolfe H. Acupuncture for Ménière's disease," Townsend Letter, vol. 244, pp. 146-147, 2003.

[26] F. H. Zhou, "Acupuncture for the treatment of 20 Ménière's syndrome," Journal of Chinese Acupuncture and Moxibustion, vol. 18, p. 26, 2002.

[27] X. J. Zhang, "Acupuncture for the treatment of 60 cases of Ménière's syndrome," Sanxi Chin Med J, vol. 24, p. 545, 2003.

[28] A. Steinberger and M. Pansini, "The treatment of Ménière's disease by acupuncture," American Journal of Chinese Medicine, vol. 11, no. 1-4, pp. 102-105, 1983.

[29] B. R. Xu and S. H. Ge, "Treatment of Ménière's disease by acupuncture: report of 75 cases," Journal of Traditional Chinese Medicine, vol. 7, no. 1, pp. 69-70, 1987.

[30] C. Tain, "Therapeutic effect of Ménière's syndrome treated by acupuncture," Chinese Journal of Acupuncture and Moxibustion, vol. 4, no. 1, pp. 5-6, 1991.

[31] J. J. Song and S. Y. Yi, "Acupuncture for the treatment of 152 cases of Ménière's syndrome," Acupunct Chin, vol. 6, p. 152, 1992.

[32] X. L. Zhu, “Treatment of Ménière's syndrome with acupuncture," Jilin Journal of Chinese Medicine, vol. 1, p. 11, 1995.

[33] L. S. Zhang and S. P. Shang, "Injection to the acupuncture points for the treatment of 286 cases of Ménière's syndrome," Shanghai Journal of Acupuncture and Moxibustion, vol. 15, p. 22, 1996.

[34] Z. H. Lu, "Ménière's syndrome treated with acupuncture: a report of 30 cases," Journal of Clinical Acupuncture, vol. 8, pp. 295-297, 1997. 
[35] D. F. Wang, "Moxibustion for the treatment of 30 cases of Ménière's syndrome," Acupunct Chin, vol. 3, p. 163, 1999.

[36] J. G. Hughes, J. Goldbart, E. Fairhurst, and K. Knowles, "Exploring acupuncturists' perceptions of treating patients with rheumatoid arthritis," Complementary Therapies in Medicine, vol. 15, no. 2, pp. 101-108, 2007.

[37] J. Yuan, D. Kerr, J. Park, X. H. Liu, and S. McDonough, "Treatment regimens of acupuncture for low back pain-a systematic review," Complementary Therapies in Medicine, vol. 16, no. 5, pp. 295-304, 2008.

[38] D. Moher, B. Pham, M. L. Lawson, and T. P. Klassen, "The inclusion of reports of randomised trials published in languages other than English in systematic reviews," Health Technology Assessment, vol. 7, pp. 1-77, 2003.

[39] K. Pilkington and J. Richardson, "Exploring the evidence: the challenges of searching for research on acupuncture," Journal of Alternative and Complementary Medicine, vol. 10, no. 3, pp. 587-590, 2004.

[40] P. G. Shekelle, S. C. Morton, M. J. Suttorp, N. Buscemi, and C. Friesen, "Challenges in systematic reviews of complementary and alternative medicine topics," Annals of Internal Medicine, vol. 142, no. 12, pp. 1042-1047, 2005.

[41] Acupuncture Regulatory Working Group, The Statutory Regulation of the Acupuncture Profession: The Report of the Acupuncture Regulatory Working Group, The Prince of Wales Foundation for Integrated Health, London, UK, 2003.

[42] A. Vickers, N. Goyal, R. Harland, and R. Rees, "Do certain countries produce only positive results? A systematic review of controlled trials," Controlled Clinical Trials, vol. 19, no. 2, pp. 159-166, 1998.

[43] H. Tsukayama and H. Yamashita, "Systematic review of clinical trials on acupuncture in the Japanese literature," Clinical Acupuncture and Oriental Medicine, vol. 3, no. 2, pp. 105-113, 2002.

[44] K. Linde, M. Scholz, D. Melchart, and S. N. Willich, "Should systematic reviews include non-randomized and uncontrolled studies? The case of acupuncture for chronic headache," Journal of Clinical Epidemiology, vol. 55, pp. 77-85, 2002.

[45] T. Wu, Q. Zhuo, J. Liu, X. Yang, and D. Moher, "Assessing the quality of Cochrane systematic reviews of Traditional Chinese Medicine," in Proceedings of the 15th Cochrane Colloquium, Sao Paulo, Brazil, October 2007. 


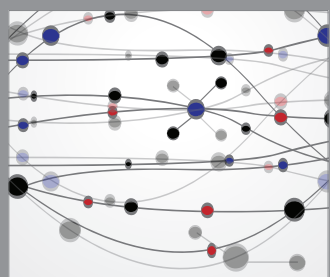

The Scientific World Journal
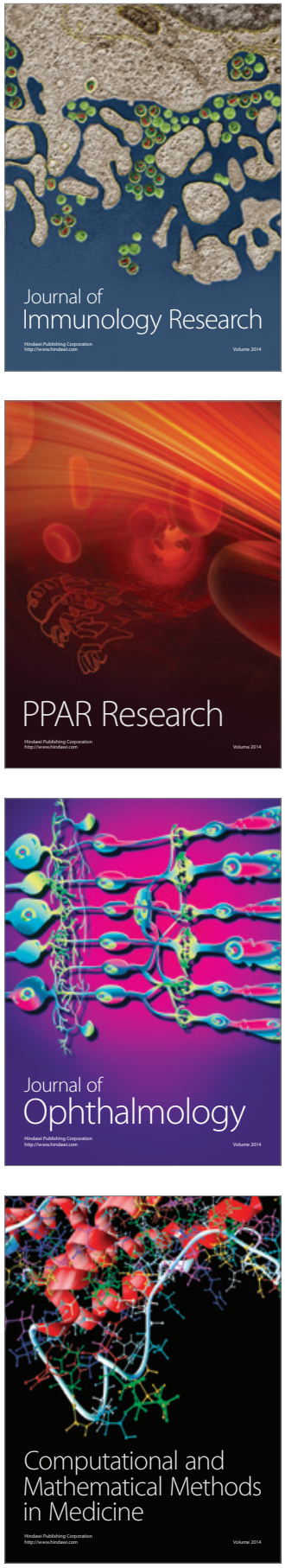

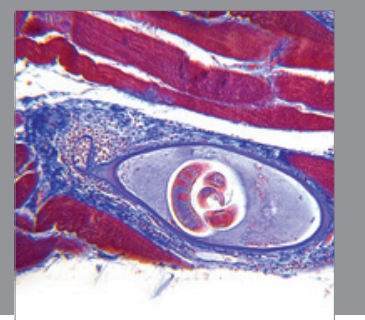

Gastroenterology

Research and Practice
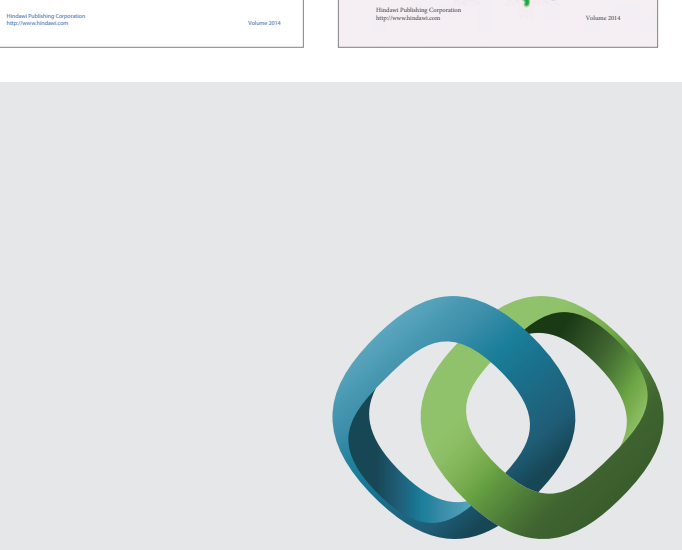

\section{Hindawi}

Submit your manuscripts at

http://www.hindawi.com
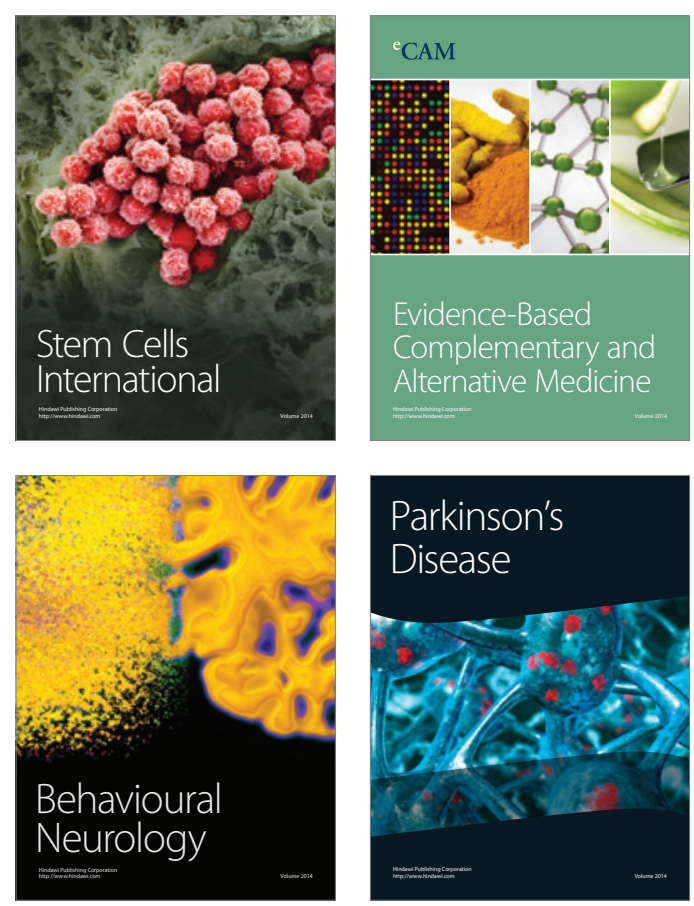

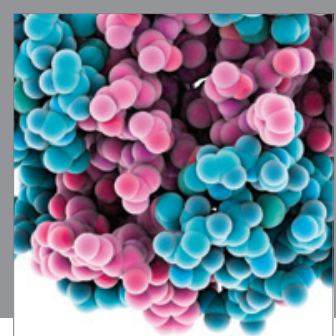

Journal of
Diabetes Research

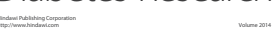

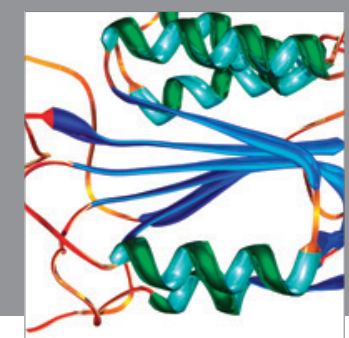

Disease Markers
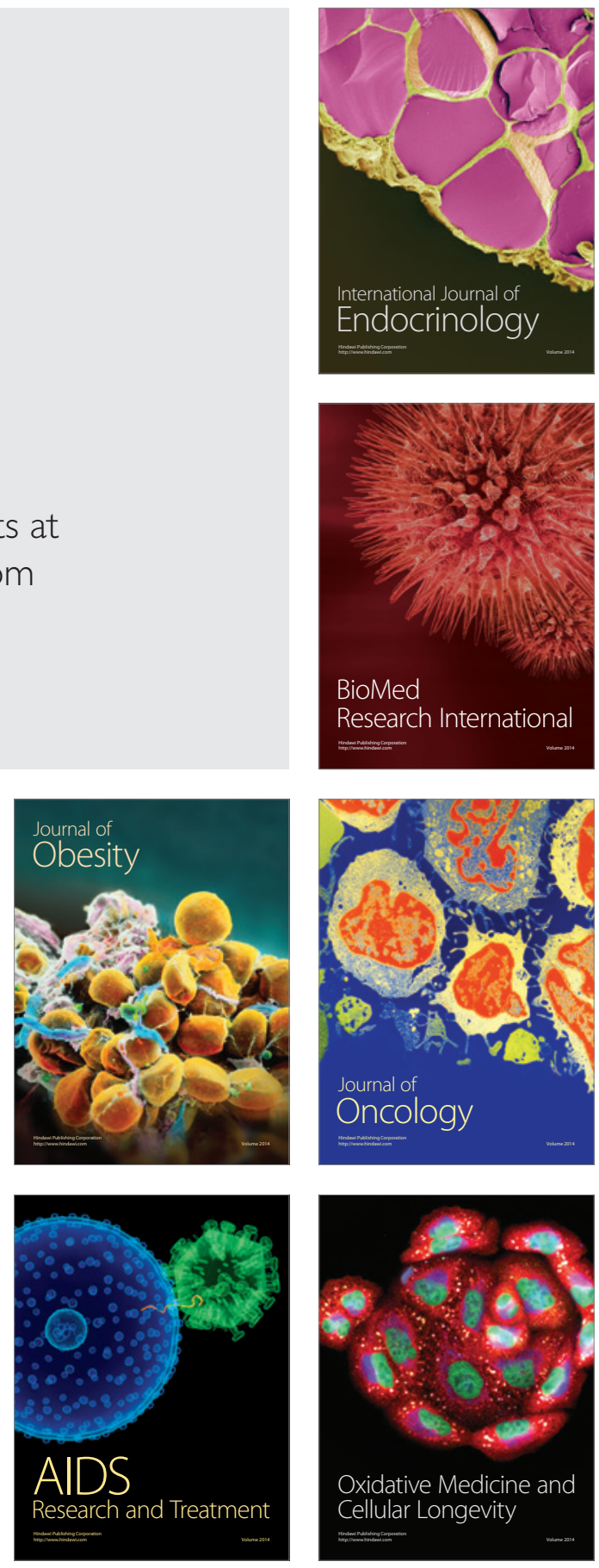